\title{
Noise study of all-normal dispersion supercontinuum sources for potential application in optical coherence tomography
}

\author{
Bravo Gonzalo, Ivan; Engelsholm, Rasmus Dybbro; Bang, Ole
}

\section{Published in:}

Proceedings of SPIE

Link to article, DOI:

$10.1117 / 12.2283060$

Publication date:

2017

Document Version

Publisher's PDF, also known as Version of record

Link back to DTU Orbit

Citation (APA):

Bravo Gonzalo, I., Engelsholm, R. D., \& Bang, O. (2017). Noise study of all-normal dispersion supercontinuum sources for potential application in optical coherence tomography. In Proceedings of SPIE (Vol. 10591).

[105910C] SPIE - International Society for Optical Engineering. Proceedings of SPIE - The International Society for Optical Engineering https://doi.org/10.1117/12.2283060

\section{General rights}

Copyright and moral rights for the publications made accessible in the public portal are retained by the authors and/or other copyright owners and it is a condition of accessing publications that users recognise and abide by the legal requirements associated with these rights.

- Users may download and print one copy of any publication from the public portal for the purpose of private study or research.

- You may not further distribute the material or use it for any profit-making activity or commercial gain

- You may freely distribute the URL identifying the publication in the public portal 


\section{Noise study of all-normal dispersion supercontinuum sources for potential application in optical coherence tomography}

I. B. Gonzalo, R. D. Engelsholm, O. Bang

I. B. Gonzalo, R. D. Engelsholm, O. Bang, "Noise study of all-normal dispersion supercontinuum sources for potential application in optical coherence tomography," Proc. SPIE 10591, 2nd Canterbury Conference on OCT with Emphasis on Broadband Optical Sources, 105910C (5 March 2018); doi: 10.1117/12.2283060

Event: Second Canterbury Conference on Optical Coherence Tomography, 2017, Canterbury, United Kingdom 


\title{
Noise study of all-normal dispersion supercontinuum sources for potential application in optical coherence tomography
}

\author{
I. B. Gonzalo*a, R. D. Engelsholm ${ }^{\mathrm{a}}$, and O. Bang, \\ ${ }^{a}$ Technical University of Denmark, DTU Fotonik, Fiber sensors and Supercontinuum, Ørsteds Plads, Kgs. Lyngby, \\ bNKT Photonics A/S, Blokken 84, DK-3460 Birkerød, Denmark \\ *ibag@ fotonik.dtu.dk
}

\section{INTRODUCTION}

Commercially available silica-fiber-based and ultra-broadband supercontinuum (SC) sources are typically generated by pumping close to the zero-dispersion wavelength (ZDW) of a photonic crystal fiber (PCF), using high-power picosecond or nanosecond laser pulses [1-3]. Despite the extremely broad bandwidths, such sources are characterized by large intensity fluctuations [2-4], limiting their performance for applications in imaging such as optical coherence tomography (OCT). An approach to eliminate the influence of noise sensitive effects is to use a so-called all-normal dispersion (ANDi) fiber, in which the dispersion is normal for all the wavelengths of interest. Pumping these types of fibers with short enough femtosecond pulses allows to suppress stimulated Raman scattering (SRS) [5], which is known to be as noisy process as modulation instability (MI) [6], and coherent SC is generated through self-phase modulation (SPM) and optical wave breaking (OWB) $[7,8]$.

In this study, we show the importance of the pump laser and fiber parameters in the design of low-noise ANDi based SC sources, for application in OCT. We numerically investigate the pulse-to-pulse fluctuations of the SC, calculating the relative intensity noise (RIN) as a function of the pump pulse duration and fiber length. Furthermore, we experimentally demonstrate the role of the fiber length on the RIN of the ANDi SC, validating the results calculated numerically. In the end, we compare the RIN of a commercial SC source based on MI and the ANDi SC source developed here, which shows better noise performance when it is carefully designed.

\section{NUMERICAL RESULTS}

The generalized Schrödinger equation (GNLSE) is solved with Matlab in order to investigate the noise performance of the SC generated in a commercially available ANDi fiber (NL-1050-NEG-1) from NKT Photonics [7]. Figure 1 (a) shows the dispersion profile and confinement loss (silica loss is also added) calculated using COMSOL Multiphysics for the fiber parameters pitch $\Lambda=1.44 \mu \mathrm{m}$ and relative hole size $d / \Lambda=0.37$. In the simulations, the input field envelope is assumed to be hyperbolic secant with peak power $44 \mathrm{~kW}$. The input noise from quantum fluctuations is added by using the one-photon-per-mode model [1,4]. An ensemble of 20 simulations with different input noise seeds is calculated along $1 \mathrm{~m}$ of fiber length for each pulse duration. The RIN is defined as the ratio of the standard deviation to the mean

$$
R I N(\omega)=\frac{\sigma(\omega)}{\mu(\omega)}=\frac{\left\langle\left(\left|\tilde{A}_{i}(\omega)\right|^{2}-\left\langle\left|\tilde{A}_{j}(\omega)\right|^{2}\right\rangle\right)^{2}\right\rangle^{1 / 2}}{\left\langle\left|\tilde{A}_{j}(\omega)\right|^{2}\right\rangle},
$$

where $\tilde{A}_{i}(\omega)$ is the field envelope of the SC. The RIN is subsequently spectrally averaged to obtain one value of the RIN at each fiber length. The spectrally averaged RIN is calculated as $[1,4]$

$$
\langle R I N\rangle=\frac{\int_{0}^{\infty} R I N(\omega)\left\langle|\tilde{A}(\omega)|^{2}\right\rangle d \omega}{\int_{0}^{\infty}\left\langle|\tilde{A}(\omega)|^{2}\right\rangle d \omega} .
$$

The angle brackets indicate ensemble average over all combinations of 20 independent SC generated with different noise seeds. The values of the RIN are between 0 (low noise) and 100\% (high noise). Figure 1 shows the evolution of the RIN for input pulses with full width at half maximum (FWHM) from 50 fs to 3 ps over $1 \mathrm{~m}$ of fiber. In contrast to [5] intensity fluctuations are investigated instead of phase fluctuations, although there is a correlation between both.

2nd Canterbury Conference on OCT with Emphasis on Broadband Optical Sources, edited by Adrian Podoleanu, Ole Bang, Proc. of SPIE Vol. 10591, 105910C

(C) 2018 SPIE · CCC code: $1605-7422 / 18 / \$ 18 \cdot$ doi: $10.1117 / 12.2283060$ 
The supercontinua generated with input pulses up to around 1 ps present low RIN, indicating that SRS is effectively suppressed [5] over $1 \mathrm{~m}$, which results in low pulse-to-pulse fluctuations. The suppression of the SRS gain is due to the nonlinear coupling between four-wave mixing (FWM) and SRS at high peak powers, as it was recently demonstrated by Heidt et al [5]. However, noisier SC is expected for extensively long fiber lengths even for short pump pulses due to the negative effect of SRS. On the other hand, using long enough pump pulses can bring SRS noise before OWB settles, and thus earlier than the completely development of the SC, resulting in a SC seeded by SRS noise. For instance, pumping with 3 ps results in high intensity fluctuations before OWB occurs as shown in Fig.1.
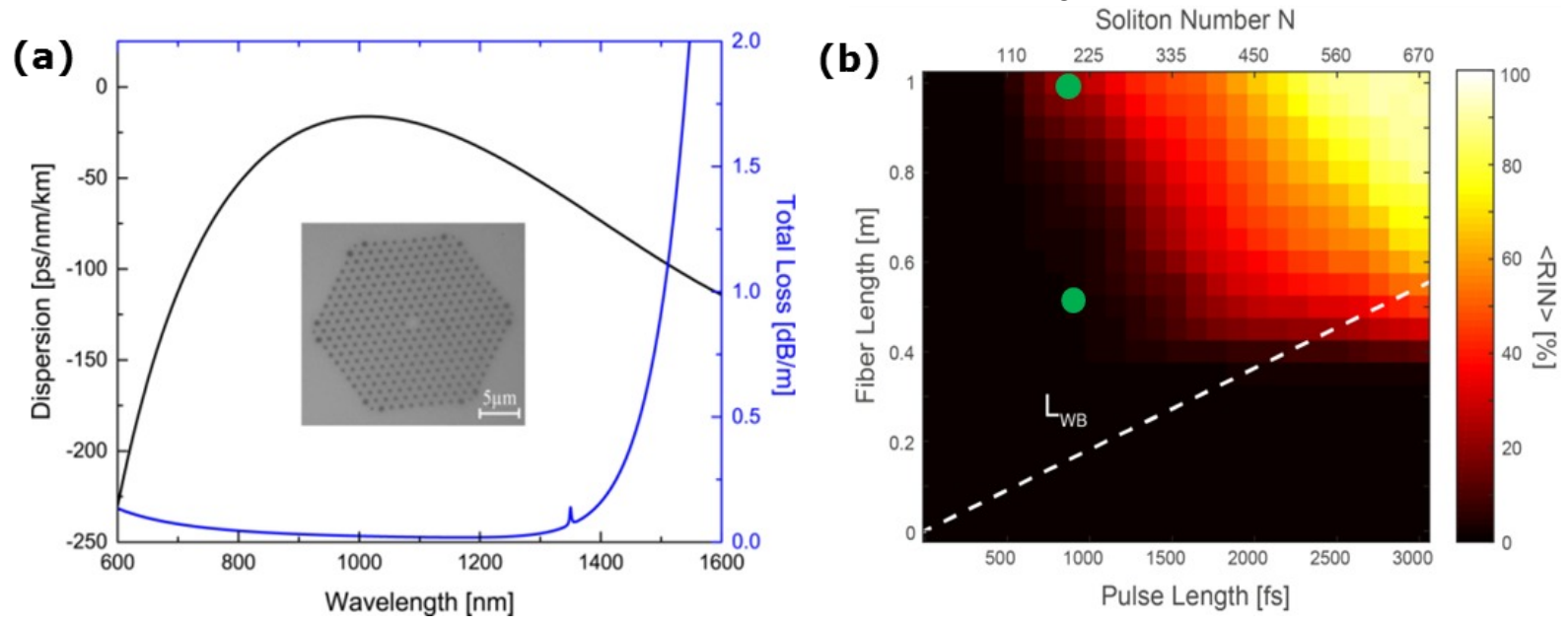

Figure 1. (a) Dispersion (black), total loss (blue) including confinement and material loss, and a microscope image of the ANDi fiber. (b) Spectrally averaged RIN of SC generated with $44 \mathrm{~kW}$ peak power for different pulse durations (FWHM) along $1 \mathrm{~m}$ of fiber. Green dots correspond to the simulation with $800 \mathrm{fs}$ after $0.5 \mathrm{~m}$ and $1 \mathrm{~m}$. LwB is the optical wave breaking distance.
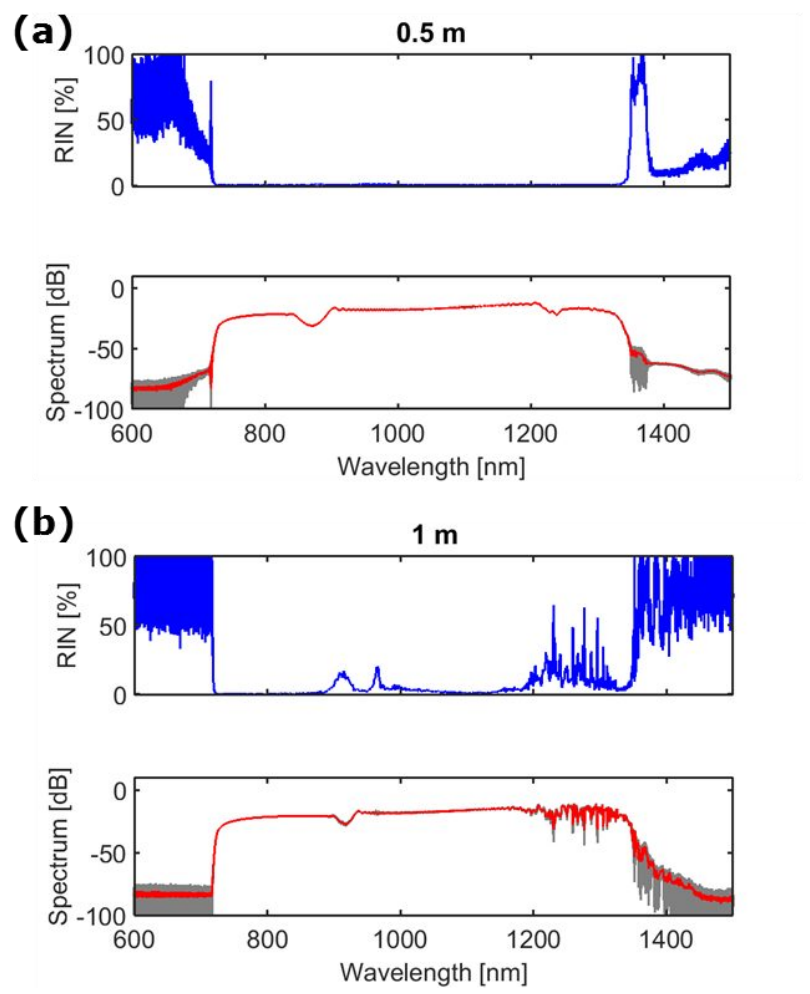

Figure 2. Spectra (red and grey) and calculated RIN (blue) corresponding to an input pulse of 800 fs and fiber length (a) 0.5 $\mathrm{m}$ and (b) $1 \mathrm{~m}$ (green dots in Fig. 1 (b)). Mean (red) and 20 shots (grey) spectra are shown for both fiber lengths. 
Therefore, careful must be taken in order to design a low-noise and coherent ANDi SC, since the phase and intensity fluctuations show up earlier in the fiber for sufficiently long pump pulses as SC is seeded by SRS noise. Moreover, even using short femtosecond pump pulses, the RIN can be high for extensively long fiber lengths, since the noise from SRS can still be generated along the fiber length as shown in Fig. 1. Figure 2 shows the SC spectra and calculated RIN for $800 \mathrm{fs}$ input pulses at two different positions in the fiber. After $0.5 \mathrm{~m}$, the SC has developed to its maximum bandwidth as the fiber length is longer than the OWB distance, which is around $0.18 \mathrm{~m}$ for the conditions in the simulation. The SC is totally coherent and the intensity fluctuations, assessed with the RIN, are negligible. However, when the SC is obtained after $1 \mathrm{~m}$ of fiber, the SC extension is the same but in this case there are intensity fluctuations in the long wavelength side and around $950 \mathrm{~nm}$. The intensity fluctuations due to SRS are noticed in the spectrum and in the calculated RIN. The SC is expected to undergo stronger intensity fluctuations if the pulses are propagated more along the fiber as seen in Fig. 1. This increase in the RIN with the propagation distance is further investigated and confirmed in the experimentally in the following section.

\section{EXPERIMENTAL RESULTS}

According to Fig. 1 (b) and Fig. 2, longer fiber lengths result in higher RIN for the same input pulse duration. This behavior is experimentally studied in this section. Figure 3 shows the experimental setup to measure the SC and intensity fluctuations of an ANDi based SC. A picosecond mode-locked laser with center wavelength $1036 \mathrm{~nm}$ and repetition rate $78 \mathrm{MHz}$ is compressed to femtosecond pulses. The autocorrelation of the compressed pulses is measured with an intensity autocorrelator (Femtochrome FR-103HP) giving 225 fs FWHM. This femtosecond laser is used to generate SC in the ANDi fiber (Fig. 1 (a)) with $0.8 \mathrm{~W}$ pump average power. The SC spectrum is recorded with an optical spectrum analyzer (ANDO AQ6317B). The intensity fluctuations are assessed in the following way. The SC is spectrally filtered every $50 \mathrm{~nm}$ [9] with $10 \mathrm{~nm}$ bandwidth bandpass filters from Thorlabs. The time series of the power through the filters is detected with two fiber-coupled photodiodes (DET08CFC - 800 to $1700 \mathrm{~nm}$ (InGaAs), $5 \mathrm{GHz}$; and DET025AFC - 400 to $1100 \mathrm{~nm}, 2 \mathrm{GHz}$ ), and then recorded with a fast oscilloscope [10] (Teledyne LeCroy - HDO9404 - 10 bits resolution, 40 $\mathrm{Gs} / \mathrm{s}$, and $4 \mathrm{GHz}$ ). The RIN value at each wavelength is obtained by measuring the maximum voltage of each pulse in the time series, subtracting the noise floor, and then calculating the ratio between standard deviation and mean. Time series of 16000 pulses were taken to do the calculations.

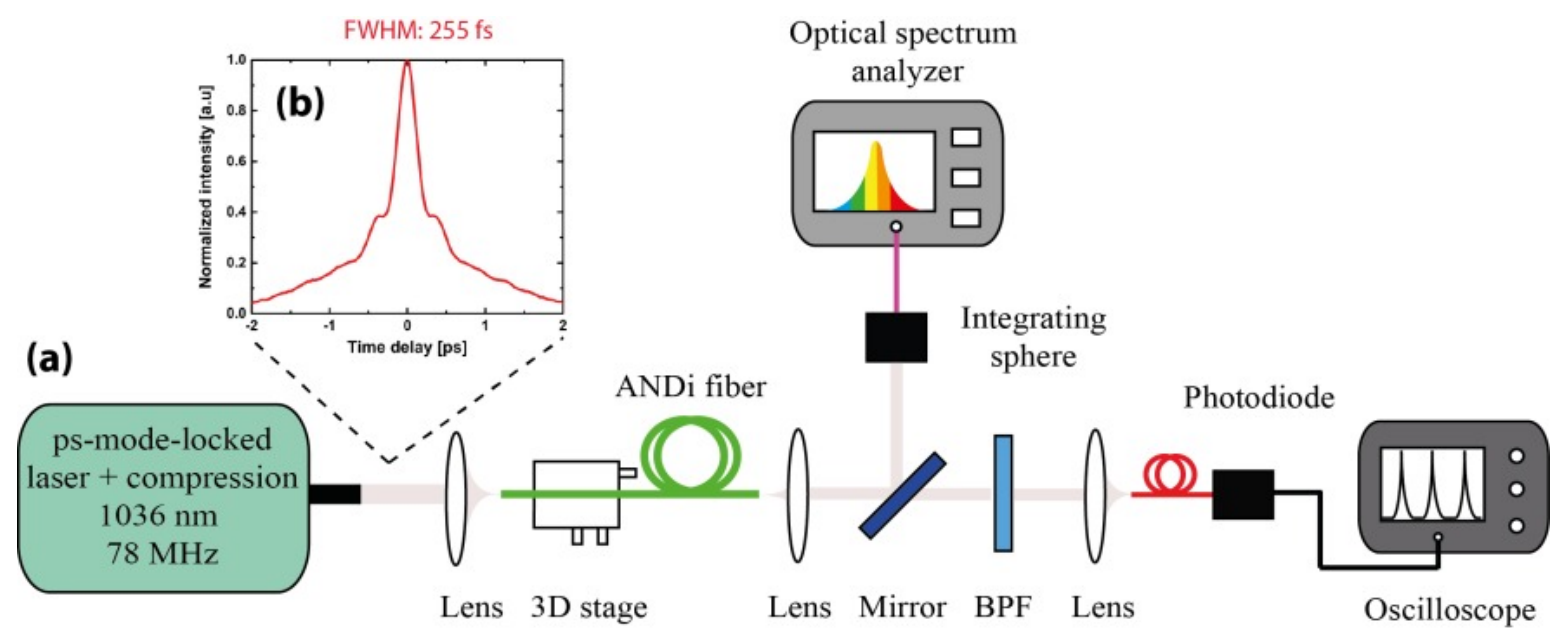

Figure 3. (a) Sketch of setup to measure supercontinuum and RIN. BPF - Bandpass filter (b) Intensity autocorrelation trace of the femtosecond laser, FWHM = 255 fs.

Figure 4 (a) and (b) shows the spectra recorded and RIN measurements of the SC generated with two different fiber lengths, $0.5 \mathrm{~m}$ and $9 \mathrm{~m}$, respectively. When a short fiber length is used, the RIN is generally low across the spectrum and thus low noise SC can be achieved with the input power and pulse duration (255 fs) used in the experiment. On the other hand, when the SC is generated with a longer fiber, in this case $9 \mathrm{~m}$, the RIN increases beyond $950 \mathrm{~nm}$, as shown in Fig. 4 (b). The longer the fiber length, the larger the intensity fluctuations in the SC spectrum due to a stronger contribution of SRS. From the simulations in Fig. 1, noise is expected to be even higher when SC is generated with longer input pulses for the same fiber length. 
The noise performance of our SC source (Fig. 4) is compared to a commercial SC source based on MI in order to evaluate the advantage of using ANDi based SC for applications where low intensity fluctuations are required. The commercial source uses high-power picosecond pulses to pump a PCF in the anomalous dispersion region, close to the $\mathrm{ZDW}$, and thus the SC is initiated by MI followed but soliton collisions and red-shift. Figure 4 (c) shows that the RIN of the MI based SC source is always higher than the ANDi based SC generated with a short fiber length. The improvement is more pronounced at longer wavelengths, where MI based SC sources are known to be noisier [2-4]. However, longer ANDi fiber lengths can produce a SC with higher intensity fluctuations than commercial sources, as seen in Fig. 4 (c). We can also see that no conclusion can be drawn in terms of noise from the flatness of the SC spectrum generated with 9 $\mathrm{m}$ since it is only due to averaging of the spectrum analyzer. Therefore, $\mathrm{SC}$ using ANDi fiber must be designed carefully to avoid SRS noise originated from long fiber lengths. The maximum fiber length that can be used to generate SC without excess noise is given by the coherence length introduced by Heidt et al, which depends on fiber parameters, pump power and pulse length [5].
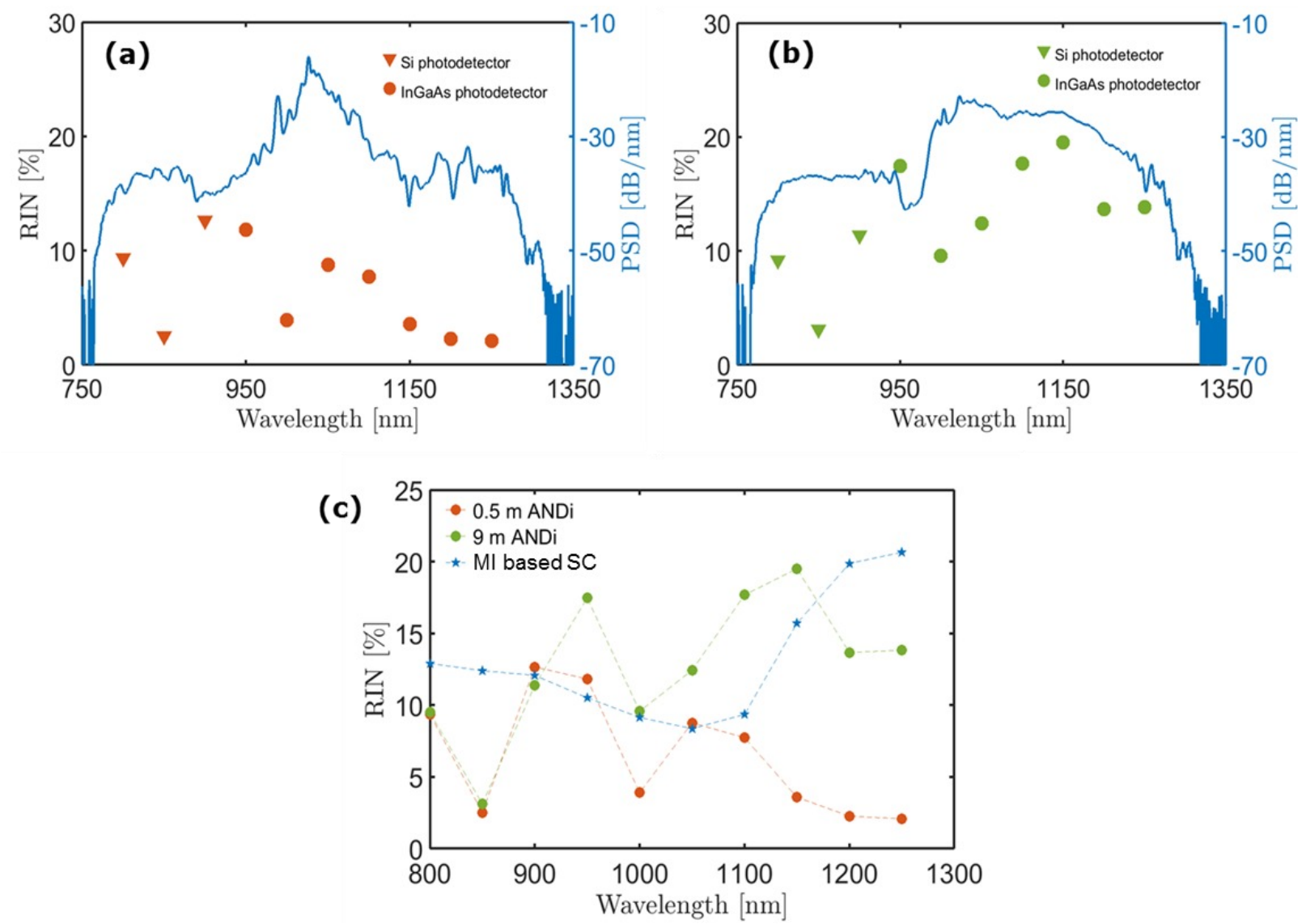

Figure 4. Measured SC spectrum (blue) and RIN for (a) $0.5 \mathrm{~m}$ (orange) and (b) $9 \mathrm{~m}$ (green) ANDi fiber lengths. (c) Comparison of the measured RIN for a MI based SC commercial source (blue) and the ANDi based SC.

\section{CONCLUSIONS}

In conclusion, we have investigated the effect of the input pulse duration and fiber length on the relative intensity noise of the SC generated with a commercially available ANDi fiber. According to the simulations, short input pulses and fiber lengths lead in general to a SC with low intensity fluctuations since the nonlinear phenomena involved are coherent. We have also shown that SC becomes noisy using excessively long fiber lengths due to SRS, as demonstrated by Heidt et al [5]. The effect of the fiber length on the SC noise was further investigated experimentally, where ANDi SC was generated with two fiber lengths. For short fiber lengths, the ANDi SC has lower intensity fluctuations than a commercial SC source based on MI. However, higher noise in the ANDi SC than MI based SC was measured when generated in a long fiber. Consequently, the choice of the pump source and ANDi fiber is crucial to achieve a good noise 
performance with these sources. When properly design, ANDi SC sources can be a promising candidate as a light source for optical coherence tomography since they can offer a broad bandwidth together with low intensity fluctuations.

\section{ACKNOWLEDGEMENTS}

The authors acknowledge support from Innovation Fund Denmark for the project ShapeOCT (J. No. 4107-00011A) and LOISE (J. No 70934).

\section{REFERENCES}

1. J. M. Dudley, G. Genty, and S. Coen, Rev. Mod. Phys. 78, 1135-1184 (2006).

2. A. Kudlinski, B. Barviau, A. Leray, C. Spriet, L. Héliot, and A. Mussot, Opt. Express 18, 27445-27454 (2010).

3. U. Møller, S. T. Sørensen, C. Jakobsen, J. Johansen, P. M. Moselund, C. L. Thomsen, and O. Bang, Opt. Express 20, 2851 (2012).

4. S. T. Sørensen, O. Bang, B. Wetzel, and J. M. Dudley, Opt. Commun. 285(9), 2451-2455 (2012).

5. A. M. Heidt, J. S. Feehan, J. H. V. Price, and T. Feurer, J. Opt. Soc. Am. B 34, 764-775 (2017).

6. U. Møller and O. Bang, Electron. Lett. 49(1), 63-65 (2013).

7. A. M. Heidt, A. Hartung, G. W. Bosman, P. Krok, E. G. Rohwer, H. Schwoerer, and H. Bartelt, Opt. Express 19, $3775-3787$ (2011).

8. M. Klimczak, G. Soboń, R. Kasztelanic, K. M. Abramski, and R. Buczyński, Scientific Reports 6, 19284 (2016).

9. K. L. Corwin, N. R. Newbury, J. M. Dudley, S. Coen, S. A. Diddams, K. Weber, and R. S. Windeler, Phys. Rev. Lett. 90, 1-4 (2003).

10. C. Lafargue, J. Bolger, G. Genty, F. Dias, J. M. Dudley, and B. J. Eggleton, IEEE Electron. Lett. 45 (2009). 